\title{
Expression of hepatocyte growth factor and $c$-Met in non-small-cell lung cancer and association with lymphangiogenesis
}

\author{
NAN ZHANG ${ }^{1 *}$, FUBO XIE $^{2 *}$, WEI GAO ${ }^{1}$, SHUWEN YU $^{3}$, LIYUN QIU $^{3}$, \\ WENLI LIN ${ }^{1}$, YUPING SUN ${ }^{1}$ and TANGHONG JIA ${ }^{4}$ \\ ${ }^{1}$ Department of Oncology, Jinan Central Hospital, Shandong University, Jinan, Shandong 250013; \\ ${ }^{2}$ Department of Radiology, Qilu Hospital, Shandong University, Jinan, Shandong 250012; \\ Departments of ${ }^{3}$ Pharmacy and ${ }^{4}$ Surgery, Jinan Central Hospital, Shandong University, \\ Jinan, Shandong 250013, P.R. China
}

Received December 16, 2013; Accepted November 7, 2014

DOI: $10.3892 / \mathrm{mmr} .2014 .3071$

\begin{abstract}
Previous experimental studies have demonstrated that hepatocyte growth factor (HGF) and its receptor $c$-Met serve an important function in lymphangiogenesis, but their biological functions in malignant tumors have remained elusive. The present study aimed to investigate the expression patterns of HGF- $\alpha$ and $c$-Met and their association with vascular endothelial growth factor (VEGF)-C, lymphatic vessel density and lymph node metastasis in non-small-cell lung cancer (NSCLC). In the present study, the lymphatic microvessel density (LMVD) and the expression levels of HGF- $\alpha$, its receptor $c$-Met and VEGF-C were determined in 113 human NSCLC tissues and 113 normal lung tissue samples, using immunohistochemical staining. As a result, it was determined that the expression levels of HGF- $\alpha, c$-Met and VEGF-C were significantly higher in NSCLC tissues than those in normal lung tissues (HGF- $\alpha, 67.3$ vs. $20.4 \%, \mathrm{P}<0.001$; $c$-Met, 74.3 vs. $23.0 \%, \mathrm{P}<0.001$; and VEGF-C, 65.5 vs. $23.9 \%$, $\mathrm{P}<0.001)$. HGF- $\alpha$ expression was observed to be significantly associated with that of VEGF-C $(r=0.234, \mathrm{P}=0.012)$ or $c$-Met $(r=0.648, \mathrm{P}<0.001)$. In addition, there was a positive correlation between the expression levels of VEGF-C and $c$-Met $(r=0.224, \mathrm{P}=0.017)$. In NSCLC tissues, the expression of HGF- $\alpha, c$-Met or VEGF-C was significantly correlated with
\end{abstract}

Correspondence to: Professor Yuping Sun, Department of Oncology, Jinan Central Hospital, Shandong University, 105 Jiefang Road, Jinan, Shandong 250013, P.R. China

E-mail: sunyuping@live.cn

Professor Tanghong Jia, Department of Surgery, Jinan Central Hospital, Shandong University, 105 Jiefang Road, Jinan, Shandong 250013, P.R. China

E-mail: jiatanghong@hotmail.com

*Contributed equally

Key words: non-small-cell lung cancer, hepatocyte growth factor, $c$-Met, lymphatic microvessel density, lymphangiogenesis, lymphatic metastasis the LMVD ( $\mathrm{P}=0.045,0.002$ and 0.001 , respectively), and lymph node metastasis was more common in HGF- $\alpha, c$-Met or VEGF-C-positive groups $(\mathrm{P}=0.020,0.020$ and 0.009 , respectively). In addition, the HGF- $\alpha$ or VEGF-C-positive groups presented shorter survival time periods. In conclusion, the expression of HGF- $\alpha$ or $c$-Met was closely correlated with VEGF-C, LMVD and metastases of lymph nodes, indicating that HGF- $\alpha, c$-Met and VEGF-C may perform important and collaborative actions in lymphangiogenesis and lymphatic metastasis of primary NSCLC.

\section{Introduction}

Primary lung cancer is one of the most common malignant tumors worldwide, with increasing levels of morbidity and mortality. Non-small-cell lung cancer (NSCLC) accounts for $75-80 \%$ of cases of primary lung cancer. NSCLC metastasis includes regional spread, lymphatic metastasis, hematogenous metastasis and airway metastasis. Lymphatic metastasis is the main route of tumor spread in NSCLC and was observed to occur when the diameter of the tumor was $<2 \mathrm{~cm}$, thus this is an important factor affecting clinical treatment and prognosis. Lymphatic metastasis is also a crucial factor in the prognosis of lung cancer (1). However, the precise mechanisms controlling lymphatic metastasis in NSCLC are not fully understood.

Lymphangiogenesis, the growth of lymphatic vessels, is a necessary process in the development of tumor metastasis (2). An increase in the number of lymphatic vessels in the tumor stroma is correlated with lymph node metastasis (3-5). Several growth factors, including vascular endothelial growth factor (VEGF), platelet-derived growth factor (PDGF), basic fibroblast growth factor (FGF), insulin-like growth factor (IGF) I/II and hepatocyte growth factor (HGF) exhibit lymphangiogenic activity (6-13). Among these growth factors, HGF and its receptor $c$-Met are frequently observed to be at high levels in the majority of types of solid tumor, and the overexpression of HGF and/or $c$-Met have also been correlated with the degree of tumor invasiveness (14-17). HGF, also known as scatter factor, can strongly stimulate the regeneration of hepatocytes (18). HGF is essential in fetal development, organ formation and tumor invasion (19-21). In addition to promoting 
tumor cell growth and invasion, HGF is also a potent hemangiogenic factor that contributes to tumor angiogenesis $(22,23)$. Thus, previous studies have indicated that HGF contributes to tumor growth and metastasis via its stimulatory effects on tumor cells and angiogenesis.

To clarify the role of HGF- $\alpha$ or $c$-Met in lymphangiogenesis in NSCLC, in the present study, immunohistochemistry was used to examine the association between the expression of HGF- $\alpha$ or $c$-Met with VEGF-C, lymphangiogenesis and various clinicopathological characteristics.

\section{Materials and methods}

Ethics statement. De-identified human tissue samples were obtained from Jinan Central Hospital, affiliated with Shandong University (Jinan, China). The use of specimens was approved by the Institutional Review Board at Jinan Central Hospital. Written informed consent was obtained from each patient in accordance with the requirements of the institution's board of ethics. The Institutional Review Board on Medical Ethics of Jinan Central Hospital approved the methods used for the collection of specimens, including written informed consent from all patients.

Patients and tissue samples. A total of 113 paraffin-embedded specimens were obtained from patients with NSCLC who underwent surgical resection between October 2009 and December 2011 at Jinan Central Hospital. None of the selected patients had undergone chemotherapy, radiotherapy or other therapies. The mean age at diagnosis was 59.72 years, with a range of 32-82 years, there were 81 males and 32 females. In total, 50 cases were adenocarcinoma, 10 were large cell carcinoma and 53 were squamous cell carcinoma. The cell differentiation degree was determined according to the latest classification, amended in 1999 (24), 87 cases of well/moderately differentiated and 26 cases of poorly differentiated cells were identified. The tumors were staged according to the USA Cancer Union Guidelines (25), 24 cases of pathological stage I, 52 stage II and 37 stage III tumors were present. All specimens were examined by two experienced pathologists. Also, 113 control specimens were selected from the corresponding adjacent normal tissues in the patients, $5 \mathrm{~cm}$ from the edge of the tumors. All samples were obtained with medical ethics approval (no. 2013018 at the Institutional Review Board on Medical Ethics of Jinan Central Hospital), and all patients provided informed consent prior to use of the specimens.

Reagents. Anti-podoplanin mouse monoclonal antibody D2-40 (\#IR072; Dako, Glostrup, Denmark), anti-HGF- $\alpha$ rabbit polyclonal immunoglobulin (Ig)G antibody (\#sc-367509; Santa Cruz Biotechnology, Inc., Dallas, TX, USA), anti-c-Met rabbit monoclonal antibody (\#ab101539; Abcam, Cambridge, UK), anti-VEGF-C rabbit polyclonal antibody (\#TA321716; OriGene Technologies, Inc., Beijing, China) and the immunohistochemical surfactant protein (SP) reagent box and diaminobenzidine (DAB) color reagent (Fuzhou Maixin Biotech Co., Ltd., Fuzhou, China).

Immunohistochemistry (IHC). IHC was conducted with the SP reagent kit according to the manufacturer's instructions.
Briefly, serial section slides of $5 \mu \mathrm{m}$ were obtained from the paraffin-embedded specimens and the paraffin medium was removed. Slides were then rehydrated by passing through serial dilutions of alcohol, then placed in citrate-EDTA ( $\mathrm{pH}$ 6.0) solution (Beyotime Institute of Biotechnology, Haimen, China) and heated at $95^{\circ} \mathrm{C}$ in a microwave oven for $10 \mathrm{~min}$. Subsequently, slides were incubated in a $3 \%$ hydrogen peroxide-methanol solution (Beyotime Institute of Biotechnology) for $10 \mathrm{~min}$ to quench endogenous peroxidases. Nonspecific binding was blocked with $10 \%$ goat serum (Huayueyang Biotechnology, Beijing, China), and slides were incubated in a humidified chamber at $4^{\circ} \mathrm{C}$ overnight with the following primary antibodies: The anti-podoplanin (1:200), anti-HGF- $\alpha$ (1:50), anti- $c$-Met (1:200) and anti-VEGF-C (1:100) antibodies. Slides were washed with phosphate-buffered saline and then incubated with streptavidin-conjugated peroxide (Merck KGaA, Darmstadt, Germany) for $30 \mathrm{~min}$. The sections were visualized by incubation with DAB (Beyotime Institute of Biotechnology) solution (0.3\% hydrogen peroxide (Jiangsu Jingshen Salt and Chemical Industry Co., Ltd., Jiangsu, China) and $0.05 \%$ DAB) and counterstained with hematoxylin (Beijing Solarbio Science \& Technology Co., Ltd., Beijing, China). Placental tissue, which is known to express high levels of HGF and $c$-Met proteins, was used as a positive control. In the negative control, the primary antibody was substituted by normal mouse IgG (Beijing Solarbio Science \& Technology Co., Ltd.).

Criteria for evaluation. The evaluation of the immunohistochemical staining of HGF- $\alpha, c$-Met and VEGF-C was performed in a double-blinded manner by two investigators simultaneously using a double-headed light microscope (CKX41; Olympus, Tokyo, Japan) without knowledge of the clinical data. In the tumor specimens, analysis of the staining was exclusively restricted to the lung tumor cell reactions. Staining of stromal cells was not considered.

As the tumors displayed heterogeneous staining, the dominant pattern was used for scoring. The mean percentage of positive-stained tumor cells was determined in at least five areas at a magnification of $\mathrm{x} 400$. A combined scoring method that accounts for the intensity of staining and the percentage of cells stained was employed, as described in a previous study (26). Strong, moderate, weak and negative staining intensities were scored as 3,2,1 and 0 , respectively. The scores indicating the percentages of positive tumor cells and staining intensities were multiplied to obtain a weighted score for each case. For example, a case with $10 \%$ weak staining, $10 \%$ moderate staining and $80 \%$ strong staining would be assigned a score of $270[(10 \times 1)+(10 \times 2)+(80 \times 3)=270)$ out of a possible score of 300 . For statistical analyses, cases with weighted scores of 0-100 were defined as negative, and all others as positive.

Lymphatic microvessel density (LMVD) was determined as previously described (27). Briefly, LMVD was measured under a light microscope (CKX41; Olympus) in a single area of invasive tumor (x200 field or $0.74 \mathrm{~mm}^{2}$ ) representative of the highest LMVD (termed the 'hot spot', the area with the most lymphatic regions). Positively stained lymphatic vessels were brownish-yellow-stained endothelial cells forming tubular structures and were situated alone or bundled, with 

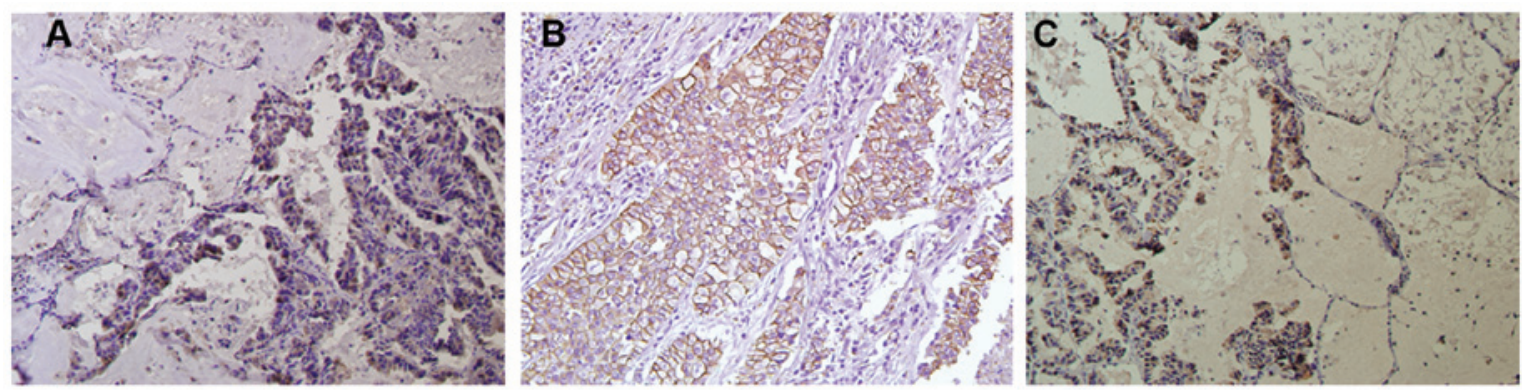

Figure 1. Cancer cells in primary NSCLC stained for HGF, $c$-Met and VEGF-C. (A) HGF-positive cancer cells in primary NSCLC with grade +++; (B) $c$-Met-positive cancer cells in primary NSCLC with grade +++; (C) VEGF-C-positive cancer cells in primary NSCLC with grade +++. Magnification, x200. NSCLC, non-small-cell lung cancer; HGF, hepatocyte growth factor; VEGF, vascular endothelial growth factor.

an open cavity inside the tubular structure. Hot spots were selected at low magnification (x40), then lymphatic vessels were counted in the five high magnification (x200) fields with the highest density. The LMVD was the median of the vessel counts in these five fields (28).

To study the association of HGF- $\alpha, c$-Met or VEGF-C with LMVD, consecutive slides from the same tumor stained for lymphatic vessels with anti-podoplanin mouse monoclonal antibody D2-40, were superimposed on the HGF- $\alpha$-, $c$-Met- or VEGF-C-stained slides. LMVD was determined in the same area. However, it was not possible to compare the LMVD in one slide that contained positive and negative areas of HGF- $\alpha$, $c$-Met or VEGF-C expression. The subjects were grouped and compared according to HGF- $\alpha$ or $c$-Met expression.

Statistical analysis. The StatView program 5.0 (Abacus Concepts, Berkeley, CA, USA) was used for all statistical analyses. Comparison of HGF- $\alpha, c$-Met or VEGF-C expression levels between NSCLC specimens and normal tissues were analyzed using the $\chi^{2}$ test. Associations between the expression of HGF- $\alpha, c$-Met and VEGF-C were analyzed by Spearman's correlation test. The association between HGF- $\alpha$, $c$-Met or VEGF-C expression and clinicopathological data was also analyzed by the $\chi^{2}$ test. Using the Mann-Whitney test, differences in distributions of LMVD in NSCLC specimens and normal lung tissues were analyzed. The association between LMVD and the expressions of HGF- $\alpha, c$-Met or VEGF-C was analyzed by Student's t-test. Survival curves were plotted according to the Kaplan-Meier method, and analyzed with the log-rank test. All statistical analyses were two-sided and $\mathrm{P}<0.05$ was considered to indicate a statistically significant difference.

\section{Results}

$H G F-\alpha$ and c-Met expression, and their association with $V E G F-C$ expression in human NSCLC tissues. Expression of HGF- $\alpha, c$-Met and VEGF-C all displayed positive cytoplasmic and/or membranous staining in NSCLC cells, with granular and heterogeneous staining in a number of the specimens (Fig. 1). $c$-Met expression was not observed in the blood or lymphatic endothelial cells in tumor tissues. Positive staining for HGF- $\alpha, c$-Met and VEGF-C was detected in the bronchial and alveolar epithelial cells in the normal specimens.

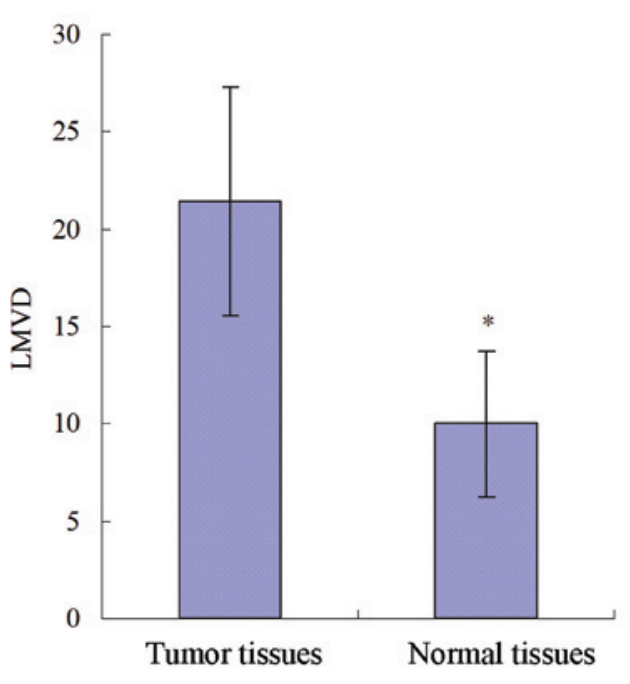

Figure 2. Quantification of the LMVD in NSCLC tissues. * $\mathrm{P}<0.05$ vs. tumor tissues. LMVD, lymphatic microvessel density.

Of the 113 patients with NSCLC, 76 (67.3\%) were positive for HGF- $\alpha, 84$ (74.3\%) for $c$-Met and 74 (65.5\%) for VEGF-C. In normal control specimens, 23 (20.4\%) were positive for HGF- $\alpha, 26$ (23.0\%) for $c$-Met and 27 (23.9\%) for VEGF-C. The expression levels of HGF- $\alpha, c$-Met and VEGF-C were significantly higher in NSCLC tissues than those in normal lung tissues $(\mathrm{P}<0.001$; Fig. 2$)$. The expression of HGF- $\alpha$ exhibited a strong positive correlation with that of $c$-Met $(\mathrm{r}=0.648$, $\mathrm{P}<0.001$ ) (Fig. 3A). In addition, HGF- $\alpha$ and $c$-Met were significantly correlated with VEGF-C expression (HGF- $\alpha, r=0.234$, $\mathrm{P}=0.012 ; c$-Met, $r=0.224, \mathrm{P}=0.017$; Fig. 3B and $\mathrm{C}$ ).

LMVD in human NSCLC specimens. Lymphatic vessels were easily identified by immunohistochemical analysis. In normal lung tissues, the lymphatic vessels were relatively small and evenly distributed. In NSCLC tissues, the lymphatic vessels were more elongated and unevenly distributed in the surrounding stroma. The LMVD in the stroma of NSCLC tissues was significantly higher than that associated with normal lung tissues $(t=6.772, \mathrm{P}<0.001$; Table I).

Association between HGF- $\alpha, c-$ Met or VEGF-C and LMVD. To assess lymphangiogenesis, IHC was conducted to observe 

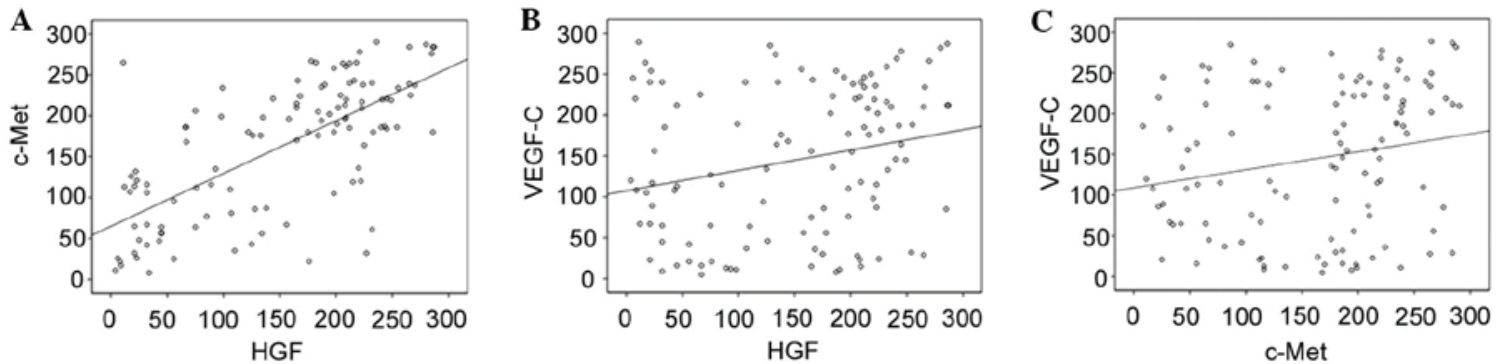

Figure 3. Association between HGF, $c$-Met and VEGF-C expression in NSCLC tissues of 113 human patients. (A) The expression of HGF exhibited a strong positive correlation with that of $c$-Met $(\mathrm{r}=0.648, \mathrm{P}<0.001)$; (B) the expression of HGF was significantly associated with VEGF-C expression $(r=0.234, \mathrm{P}=0.012)$; and $(C)$ the expression of $c$-Met was significantly associated with VEGF-C expression $(r=0.224, \mathrm{P}=0.017)$. Correlations were analyzed by Spearman's rank correlation test. HGF, hepatocyte growth factor; VEGF, vascular endothelial growth factor; NSCLC, non-small-cell lung cancer.
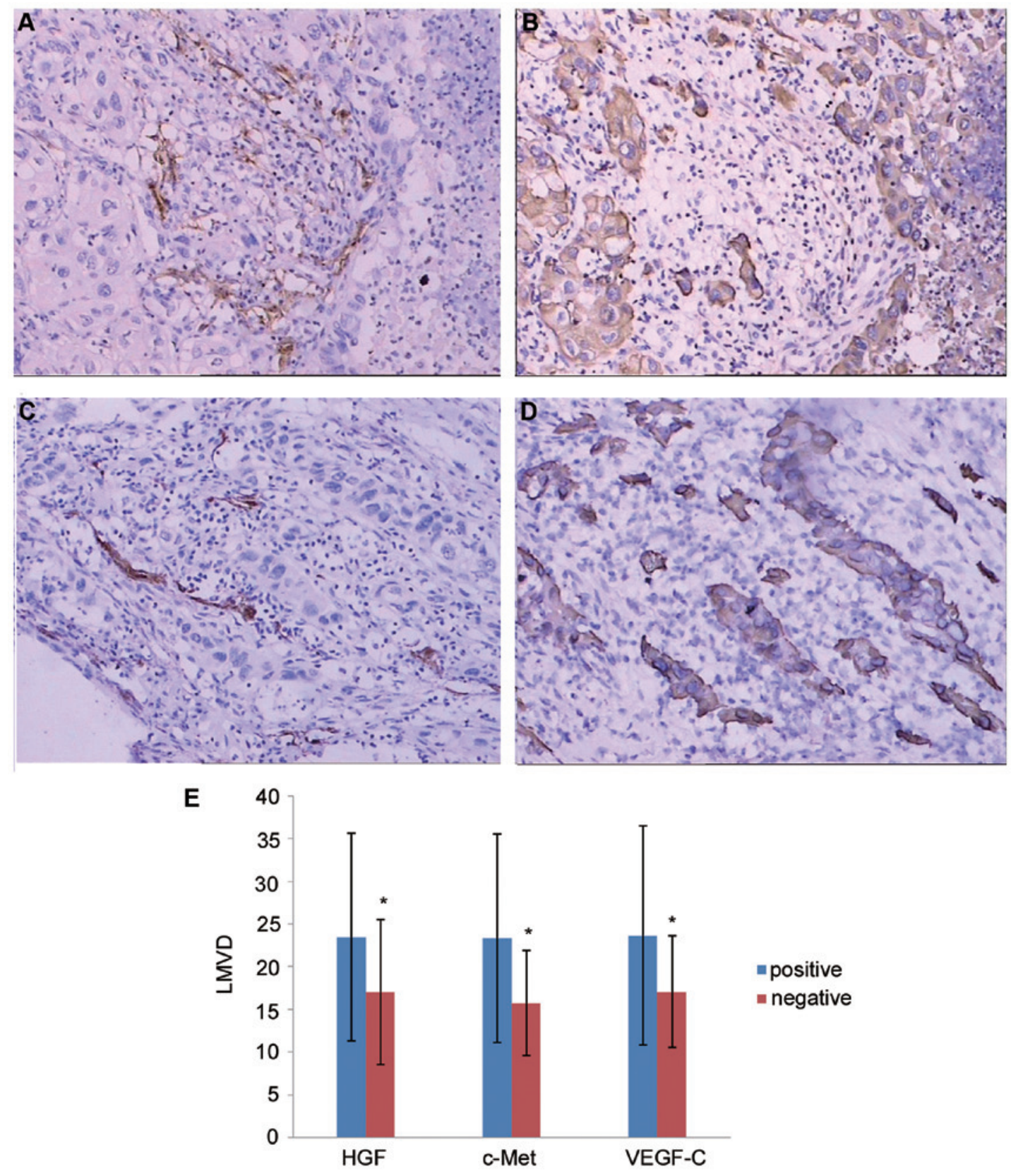

Figure 4. Association between HGF, $c$-Met or VEGF-C and LMVD in human NSCLC tissues. (A) HGF-positive cancer cells in primary NSCLC with grade +++; (B) IHC displaying LMVD intensity in consecutive slides from the tumor used in (A); (C) $c$-Met-positive cancer cells in primary NSCLC with grade +++; (D) IHC displaying LMVD intensity in consecutive slides from the tumor used in (C); magnification, x100. (E) Quantification of the LMVDs in the different groups. " $\mathrm{P}<0.05$ vs. negative expression. HGF, hepatocyte growth factor; VEGF, vascular endothelial growth factor; LMVD, lymphatic microvessel density; NSCLC, non-small-cell lung cancer; IHC, immunohistochemistry. 
Table I. HGF, $c$-Met and VEGF-C expression in 113 human NSCLC tissues.

\begin{tabular}{|c|c|c|c|c|c|c|}
\hline \multirow[b]{2}{*}{ Tissue } & \multicolumn{2}{|c|}{ HGF } & \multicolumn{2}{|c|}{$c$-Met } & \multicolumn{2}{|c|}{ VEGF-C } \\
\hline & Positive & Negative & Positive & Negative & Positive & Negative \\
\hline Tumor & 76 & 37 & 84 & 29 & 74 & 39 \\
\hline Normal & 23 & 90 & 26 & 87 & 27 & 86 \\
\hline P-value & \multicolumn{2}{|c|}{0.000} & \multicolumn{2}{|c|}{0.000} & \multicolumn{2}{|c|}{0.000} \\
\hline
\end{tabular}

HGF, hepatocyte growth factor; VEGF, vascular endothelial growth factor.

Table II. Association between expression of HGF, $c$-Met or VEGF-C and clinicopathologic factors.

\begin{tabular}{|c|c|c|c|c|c|c|}
\hline \multirow[b]{2}{*}{ Clinical feature } & \multicolumn{2}{|c|}{ HGF } & \multicolumn{2}{|c|}{$c$-Met } & \multicolumn{2}{|c|}{ VEGF-C } \\
\hline & Positive & Negative & Positive & Negative & Positive & Negative \\
\hline \multicolumn{7}{|l|}{ Age (years) } \\
\hline$\geq 65$ & 57 & 29 & 63 & 23 & 54 & 32 \\
\hline$<65$ & 19 & 8 & 21 & 6 & 20 & 7 \\
\hline P-value & \multicolumn{2}{|c|}{0.693} & \multicolumn{2}{|c|}{0.639} & \multicolumn{2}{|c|}{0.282} \\
\hline \multicolumn{7}{|l|}{ Gender } \\
\hline Male & 51 & 30 & 60 & 21 & 54 & 27 \\
\hline Female & 25 & 7 & 24 & 8 & 20 & 12 \\
\hline P-value & \multicolumn{2}{|c|}{0.122} & \multicolumn{2}{|c|}{0.919} & \multicolumn{2}{|c|}{0.675} \\
\hline \multicolumn{7}{|l|}{ T stage } \\
\hline T1-2 & 32 & 17 & 35 & 14 & 33 & 16 \\
\hline T3-4 & 44 & 20 & 49 & 15 & 41 & 23 \\
\hline P-value & \multicolumn{2}{|c|}{0.699} & \multicolumn{2}{|c|}{0.536} & \multicolumn{2}{|c|}{0.716} \\
\hline \multicolumn{7}{|l|}{$\mathrm{N}$ stage } \\
\hline No & 38 & 27 & 43 & 22 & 36 & 29 \\
\hline $\mathrm{N} 1-2$ & 38 & 10 & 41 & 7 & 38 & 10 \\
\hline P-value & \multicolumn{2}{|c|}{$0.02^{\mathrm{a}}$} & \multicolumn{2}{|c|}{$0.02^{\mathrm{a}}$} & \multicolumn{2}{|c|}{$0.009^{\mathrm{a}}$} \\
\hline \multicolumn{7}{|l|}{ Histological type } \\
\hline Squamous carcinoma & 42 & 11 & 44 & 9 & 35 & 18 \\
\hline Adenocacinoma & 27 & 23 & 32 & 18 & 30 & 20 \\
\hline Large cell carcinoma & 7 & 3 & 8 & 2 & 9 & 1 \\
\hline P-value & \multicolumn{2}{|c|}{$0.024^{\mathrm{a}}$} & \multicolumn{2}{|c|}{0.08} & \multicolumn{2}{|c|}{0.189} \\
\hline \multicolumn{7}{|l|}{ Differentiation grade } \\
\hline Well/moderately & 55 & 32 & 64 & 23 & 55 & 32 \\
\hline Poorly & 21 & 5 & 20 & 6 & 19 & 7 \\
\hline P-value & \multicolumn{2}{|c|}{0.094} & \multicolumn{2}{|c|}{0.731} & \multicolumn{2}{|c|}{0.354} \\
\hline \multicolumn{7}{|l|}{ TNM stage } \\
\hline I & 10 & 14 & 13 & 11 & 14 & 10 \\
\hline II & 38 & 14 & 41 & 11 & 28 & 24 \\
\hline III & 28 & 9 & 30 & 7 & 32 & 5 \\
\hline P-value & \multicolumn{2}{|c|}{$0.01^{\mathrm{a}}$} & & & & \\
\hline
\end{tabular}

${ }^{\mathrm{a}} \mathrm{P}<0.05$ indicates a significant correlation. TNM, tumor node metastasis; VEGF, vascular endothelial growth factor; HGF, hepatocyte growth factor. 

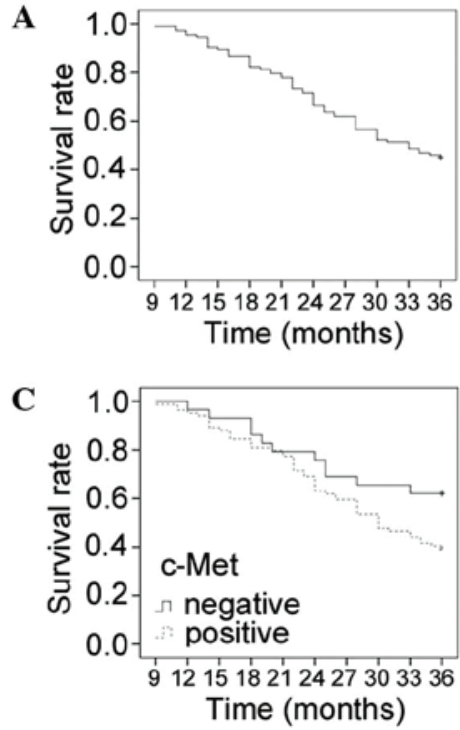
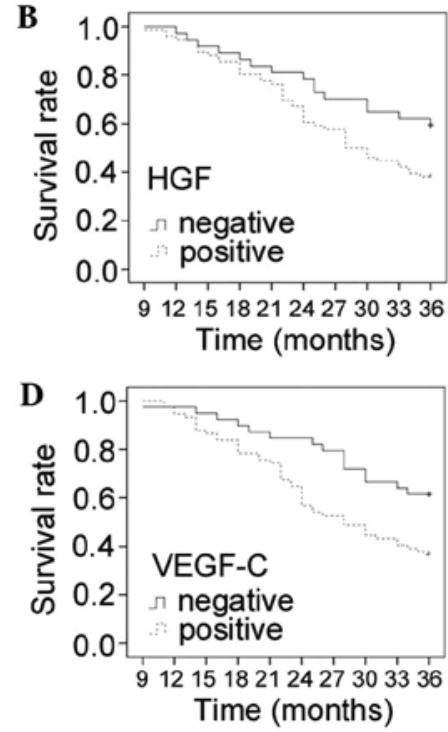

Figure 5. Kaplan-Meier analysis of the overall survival in different patient groups. (A) The overall three-year survival rate of all 113 patients was $45.1 \%$; (B) Patients with negative and positive expression of HGF (59.5 vs. 38.2\%; $\mathrm{P}=0.042)$; (C) patients with negative and positive expression of $c$-Met (62.1 vs. $39.3 \%$; $\mathrm{P}=0.063$ ); and (D) patients with negative and positive expression of VEGF-C (61.5 vs. $36.5 \%$; $\mathrm{P}=0.010)$. HGF, hepatocyte growth factor; VEGF, vascular endothelial growth factor.

the association between the expression of the above factors with the LMVD (Fig. 4A-D). It was observed that the LMVD was $23.5224 \pm 12.16362$ in HGF- $\alpha$-positive specimens, and $17.0703 \pm 8.51361$ in HGF- $\alpha$-negative specimens (Fig. 4E). The LMVD was significantly higher in the HGF- $\alpha$-positive specimens compared with those that were HGF- $\alpha$-negative $(t=3.265, \mathrm{P}=0.045)$. Similar patterns were also observed in specimens with positive $c$-Met or VEGF-C expression. They presented significantly higher LMVDs than those with negative $c$-Met or VEGF-C expression $(23.3619 \pm 12.23578$ vs. $15.7552 \pm 6.17649, t=4.322, \mathrm{P}=0.002 ; 23.6622 \pm 12.83703$ vs $17.1359 \pm 6.53817, t=3.580, \mathrm{P}=0.001$; Fig. $4 \mathrm{E})$.

Association between expression of $H G F-\alpha, c-M e t$ or VEGF-C and clinicopathological factors. A comparison between positive and negative expression of the three molecules was conducted in order to examine the potential association with clinicopathological characteristics. In 48 NSCLC patients with lymph node metastases (stage N1-2), 38 had HGF- $\alpha$-positive, $41 c$-Met-positive and 38 VEGF-C-positive tumors (Table I). In the 65 NSCLC patients without lymph node metastases (N0), 38 had HGF- $\alpha$-positive, $43 c$-Met-positive and 36 VEGF-C-positive tumors. Positive expression of HGF- $\alpha$, $c$-Met or VEGF-C was significantly associated with lymph node metastases $(\mathrm{P}=0.020, \mathrm{P}=0.020$ and $\mathrm{P}=0.009$, respectively).

Positive expression of HGF- $\alpha, c$-Met or VEGF-C was correlated with tumor node metastasis (TNM) stages $(\mathrm{P}=0.010$, $\mathrm{P}=0.038$ or $\mathrm{P}=0.004$, respectively). Adenocarcinoma and large cell carcinoma were considered as one group (non-squamous carcinoma) to be compared with the squamous carcinoma group and it was found that HGF- $\alpha$ expression was much higher in the non-squamous carcinoma specimens $(\mathrm{P}=0.024)$.

No significant association was identified between HGF- $\alpha, c$-Met or VEGF-C and any other clinicopathological factors (Table II).
Overall survival in patients with NSCLC. The three-year overall survival rate of all the 113 patients was $45.1 \%$. Fig. 5 presents the Kaplan-Meier curves for patients with positive or negative expression of HGF- $\alpha$ (Fig. 5B), $c$-Met (Fig. 5C) or VEGF-C (Fig. 5D). The overall three-year survival rates of patients positive for HGF- $\alpha$ or VEGF-C were significantly lower than the rates in the corresponding patients with negative expression ( 38.2 vs. $59.5 \%, \mathrm{P}=0.042$; or 36.5 vs. $61.5 \%$, $\mathrm{P}=0.010$, respectively). However, no significant difference in survival times was observed between the $c$-Met-positive and -negative groups (62.1\% vs. $39.3 \%$; $\mathrm{P}=0.063)$.

\section{Discussion}

HGF, first obtained from purified plasma and platelets in partially resected rat liver, is able to stimulate hepatocyte proliferation. It has also been demonstrated that a diffusion factor termed scatter factor, which is secreted by fibroblasts and is able to induce scattering in epithelial cells, is the same as HGF (18). $c$-Met, a proto-oncogene that contains a tyrosine kinase domain, initiates a range of signals to regulate cellular functions (17). A previous study indicated that, mediated by its receptor $c$-Met, HGF is able to promote proliferation, migration and angiogenesis in various types of tumor and is also important in tumoral invasion and metastasis. Overexpression of HGF and/or $c$-Met has been reported in various types of human cancer, including NSCLC and breast cancer $(29,30)$.

In the present study, the expression of HGF- $\alpha$ or $c$-Met was identified to be significantly higher in NSCLC tissues than in normal lung tissues, and it was associated with VEGF-C expression. In addition, HGF- $\alpha, c$-Met and VEGF-C expression were all associated with lymphatic metastasis in NSCLC, which suggests that HGF- $\alpha$ and $c$-Met may facilitate tumoral lymph node metastasis. Furthermore, a connection between 
the positive expression of HGF- $\alpha$ or VEGF-C and shorter survival times in patients with NSCLC were observed. Other clinical studies of the $\mathrm{HGF} / c$-Met pathway in NSCLC have also demonstrated an association with poor survival rates of NSCLC patients (31-34). These findings, taken together, suggest that HGF- $\alpha, c$-Met and VEGFC may serve as prognostic biomarkers in NSCLC.

Previous studies have suggested that HGF and $c$-Met are enhancers of tumor development, as they promote tumor cell growth, invasion, angiogenesis (29) and lymphangiogenesis (35). However, the underlying mechanisms by which HGF and $c$-Met are involved in NSCLC progression have remained elusive. Hence, the present study investigated the association of HGF or $c$-Met with lymphangiogenesis using IHC on samples from patients with NSCLC.

In the present study, LMVD was used as an indicator of lymphangiogenesis. The podoplanin antibody (D2-40), a sensitive marker that distinguishes lymphatic vessels from vascular vessels, was used to measure LMVD in adjacent stroma (36). LMVD was identified as significantly associated with lymph node metastasis and other clinicopathological factors (28). However, a previous study suggested that lymphangiogenesis may not be an important factor in lymph node metastasis in NSCLC, hence this subject requires further study (37). In the present study, the lymphatic vessels in NSCLC tissues were more elongated and unevenly distributed in the surrounding stroma, and the LMVD was significantly higher in the cancer tissues than in the normal lung tissues, making it easier for invasive tumor cells to enter the lymphatic system and thereby be transported to regional lymph nodes. These findings are consistent with those of previous studies $(4,28)$. Another study demonstrated that the center of tumors do not contain functional lymphatic vessels; however, the lymphatic vessels at the tumor margins do facilitate the lymphatic spread of tumor cells (38).

The lymphatic system is essential for the maintenance of normal functions, such as the internal environment and immune surveillance (39). Similar to blood vessels, the lymphatic vasculature remains quiescent under physiological conditions, with the exception of pathological conditions such as tumor growth and metastasis. Lymphatic metastasis is the predominant route for tumor spread and consists of a complex process with a few detailed steps. The process of lymphangiogenesis may be regulated by multiple growth factors, including VEGF, FGF, PDGF, HGF and IGF families (6-13); In the present study, cancer tissues with VEGF-C, HGF- $\alpha$ or $c$-Met-positive expression presented a significantly increased LMVD, which reconfirms that VEGF-C, HGF and $c$-Met may be able to promote lymphangiogenesis. Experiments in prostate and breast xenotranplant tumor models in nude mice have suggested that the expression of podoplanin and lymphatic vessel endothelial hyaluronan receptor-1 in tumors can be increased by the injection of recombinant human $\mathrm{HGF}$. This effect may be canceled by $\mathrm{NK} 4$, the antagonist of $\mathrm{HGF}$, indicating that HGF may promote lymphangiogenesis directly (18). A number of studies have indicated that lymphatic endothelial cells cultured in vitro express $c$-Met, and the proliferation of lymphatic endothelial cells is promoted by stimulation with HGF, which strengthens the theory that HGF may promote lymphangiogenesis directly (40). In the present study, the co-expression of HGF- $\alpha$ and $c$-Met in NSCLC was observed, and it was associated with LMVD and lymph node metastasis, but no expression in lymphatic vessels was detected. Accordingly, it was hypothesized that HGF may promote lymphangiogenesis and lymph node metastasis in tumors indirectly through an alternative pathway.

The best-studied lymphangiogenic signaling system in cancer is the VEGF-C/VEGF-D/VEGFR-3 signaling pathway. Activated by its ligands VEGF-C and VEGF-D, VEGFR-3 may lead to proliferation of lymphatic endothelial cells and the growth of lymphatic vessels (16). Cao et al (35) identified that HGF can induce lymphangiogenesis, which may be partly inhibited by soluble VEGFR-3; thus, HGF may promote lymphangiogenesis indirectly via VEGFR-3. In the present study, it was also observed that the expression of HGF- $\alpha$ or $c$-Met was associated with VEGF-C expression in NSCLC tissues. A previous study demonstrated that FGF-2 and VEGF-C, two lymphangiogenic factors, collaboratively promote angiogenesis and lymphangiogenesis in the tumor microenviroment, leading to widespread pulmonary and lymph node metastases (41). Similarly, another study demonstrated that the VEGF-A/HGF combination was able to induce a strong angiogenic response and the expression of prospero homeobox protein 1 in the lymphatic endothelial cells of the chick embryo chorioallantoic membrane (42). Thus, cancer metastasis is a complex process that engages various cytokines and their cross-talk. Further studies are therefore required to define the association of HGF and the VEGF-C/VEGF-D/VEGFR-3 or other pathways in human tumors.

The present study demonstrated that HGF- $\alpha$ and $c$-Met were highly expressed in NSCLC tissues, and associated with VEGF-C, LMVD and lymphatic metastasis, suggesting that $\mathrm{HGF}-\alpha / c-$ Met and VEGF-C may be collaboratively responsible for the induction of lymphangiogenesis in NSCLC. Of note, HGF- $\alpha$ and VEGF-C expression were observed to be associated with poor survival. These findings have implications for the targeting of HGF- $\alpha$ and $c$-Met for the therapeutic blockage of lymphangiogenesis and lymphatic metastasis.

\section{Acknowledgements}

The present study was supported by Shandong Province Natural Scientific Fund (grant no. ZR2011HM070).

\section{References}

1. Kotoulas CS, Foroulis CN, Kostikas K, et al: Involvement of lymphatic metastatic spread in non-small cell lung cancer accordingly to the primary cancer location. Lung Cancer 44: 183-191, 2004.

2. Stacker SA, Achen MG, Jussila L, Baldwin ME and Alitalo K: Lymphangiogenesis and cancer metastasis. Nat Rev Cancer 2: 573-583, 2002.

3. Franchi A, Gallo O, Massi D, Baroni G and Santucci M: Tumor lymphangiogenesis in head and neck squamous cell carcinoma: a morphometric study with clinical correlations. Cancer 101: 973-978, 2004.

4. Dadras SS, Lange-Asschenfeldt B, Velasco P, et al: Tumor lymphangiogenesis predicts melanoma metastasis to sentinel lymph nodes. Mod Pathol 18: 1232-1242, 2005.

5. Gombos Z, Xu X, Chu CS, Zhang PJ and Acs G: Peritumoral lymphatic vessel density and vascular endothelial growth factor $\mathrm{C}$ expression in early-stage squamous cell carcinoma of the uterine cervix. Clin Cancer Res 11: 8364-8371, 2005. 
6. Chen SX,LiXY, Kong XL and Feng Y: The expression of vascular endothelial growth factor- $\mathrm{C}$ in oral squamous cell carcinoma and its association with angiogenesis, lymphangiogenesis and lymph node metastasis. West China Journal of Stomatology 28 : 319-323, 2010 (In Chinese)

7. Kubo H, Cao R, Brakenhielm E, et al: Blockade of vascular endothelial growth factor receptor-3 signaling inhibits fibroblast growth factor-2-induced lymphangiogenesis in mouse cornea. Proc Natl Acad Sci USA 99: 8868-8873, 2002.

8. Vincent L and Rafii S: Vascular frontiers without borders: multifaceted roles of platelet-derived growth factor (PDGF) in supporting postnatal angiogenesis and lymphangiogenesis. Cancer Cell 6: 307-309, 2004.

9. Kajiya K, Hirakawa S, Ma B, Drinnenberg I and Detmar M Hepatocyte growth factor promotes lymphatic vessel formation and function. EMBO J 24: 2885-2895, 2005.

10. Björndahl M, Cao R, Nissen LJ, et al: Insulin-like growth factors 1 and 2 induce lymphangiogenesis in vivo. Proc Nat Acad Sci USA 102: 15593-15598, 2005

11. Sedivy R, Beck-Mannagetta J, Haverkampf C, Battistutti W and Hönigschnabl S: Expression of vascular endothelial growth factor-C correlates with the lymphatic microvessel density and the nodal status in oral squamous cell cancer. J Oral Pathol Med 32: 455-460, 2003

12. Swoboda A, Schanab O, Tauber S, et al: MET expression in melanoma correlates with a lymphangiogenic phenotype. Hum Mol Genet 21: 3387-3396, 2012.

13. Kim CH, Moon SK, Bae JH, et al: Expression of hepatocyte growth factor and c-Met in hypopharyngeal squamous cell carcinoma. Acta Otolaryngol 126: 88-94, 2006.

14. Amemiya H, Kono K, Itakura J, et al: c-Met expression in gastric cancer with liver metastasis. Oncology 63: 286-296, 2002.

15. Jedeszko C, Victor BC, Podgorski I and Sloane BF: Fibroblast hepatocyte growth factor promotes invasion of human mammary ductal carcinoma in situ. Cancer Res 69: 9148-9155, 2009.

16. Knudsen BS, Gmyrek GA, Inra J, et al: High expression of the Met receptor in prostate cancer metastasis to bone. Urology 60: 1113-1117, 2002.

17. Takeuchi H, Bilchik A, Saha S, et al: c-MET expression level in primary colon cancer: a predictor of tumor invasion and lymph node metastases. Clin Cancer Res 9: 1480-1488, 2003.

18. Jiang W, Hiscox S, Matsumoto K and Nakamura T: Hepatocyte growth factor/scatter factor, its molecular, cellular and clinical implications in cancer. Crit Rev Oncol Hematol 29: 209-248, 1999.

19. Gentile A and Comoglio PM: Invasive growth: a genetic program Int J Dev Biol 48: 451-456, 2004

20. Trusolino L and Comoglio PM: Scatter-factor and semaphorin receptors: cell signalling for invasive growth. Nat Rev Cancer 2 289-300, 2002

21. Salgia R: Role of c-Met in cancer: emphasis on lung cancer. Semin Oncol 36 (Suppl 1): S52-S58, 2009.

22. Davies G, Mason MD, Martin TA, et al: The HGF/SF antagonist NK4 reverses fibroblast- and HGF-induced prostate tumor growth and angiogenesis in vivo. Int J Cancer 106: 348-354, 2003.

23. You WK and McDonald DM: The hepatocyte growth factor/c-Met signaling pathway as a therapeutic target to inhibit angiogenesis BMB Rep 41: 833-839, 2008.

24. Fleming ID, Phillips JL, Menck HR, Murphy GP and Winchester DP: The National Cancer Data Base report on recent hospital cancer program progress toward complete American Joint Committee on Cancer/TNM staging. Cancer 80: $2305-2310,1997$.
25. Hensing TA: Clinical evaluation and staging of patients who have lung cancer. Hematol Oncol Clin North Am 19: 219-235, 2005.

26. Hasina R, Whipple ME, Martin LE, et al: Angiogenic heterogeneity in head and neck squamous cell carcinoma: biological and therapeutic implications. Lab Invest 88: 342-353, 2008

27. Weidner N: Current pathologic methods for measuring intratumoral microvessel density within breast carcinoma and other solid tumors. Breast Cancer Res Treat 36: 169-180, 1995.

28. Adachi Y, Nakamura H, Kitamura Y, et al: Lymphatic vessel density in pulmonary adenocarcinoma immunohistochemically evaluated with anti-podoplanin or anti-D2-40 antibody is correlated with lymphatic invasion or lymph node metastases. Pathol Int 57: 171-177, 2007.

29. Olivero M, Rizzo M, Madeddu R, et al: Overexpression and activation of hepatocyte growth factor/scatter factor in human non-small-cell lung carcinomas. Br J Cancer 74: 1862-1868, 1996.

30. Edakuni G, Sasatomi E, Satoh T, Tokunaga O and Miyazaki K: Expression of the hepatocyte growth factor/c-Met pathway is increased at the cancer front in breast carcinoma. Pathol Int 51: $172-178,2001$.

31. Ichimura E, Maeshima A, Nakajima $T$ and Nakamura $T$ : Expression of c-met/HGF receptor in human non-small cell lung carcinomas in vitro and in vivo and its prognostic significance. Jpn J Cancer Res 87: 1063-1069, 1996.

32. Takanami I, Tanana F, Hashizume T, et al: Hepatocyte growth factor and c-Met/hepatocyte growth factor receptor in pulmonary adenocarcinomas: an evaluation of their expression as prognostic markers. Oncology 53: 392-397, 1996.

33. Siegfried JM, Weissfeld LA, Singh-Kaw P, et al: Association of immunoreactive hepatocyte growth factor with poor survival in resectable non-small cell lung cancer. Cancer Res 57: 433-439, 1997.

34. Toschi L and Cappuzzo F: Clinical implications of MET gene copy number in lung cancer. Future Oncol 6: 239-247, 2010.

35. Cao R, Björndahl MA, Gallego MI, et al: Hepatocyte growth factor is a lymphangiogenic factor with an indirect mechanism of action. Blood 107: 3531-3536, 2006.

36. Kahn HJ, Bailey D and Marks A: Monoclonal antibody D2-40, a new marker of lymphatic endothelium, reacts with Kaposi's sarcoma and a subset of angiosarcomas. Mod Pathol 15: 434-440, 2002.

37. Faoro L, Hutto JY, Salgia R, et al: Lymphatic vessel density is not associated with lymph node metastasis in non-small cell lung carcinoma. Arch Pathol Lab Med 132: 1882-1888, 2008.

38. Padera TP, Kadambi A, di Tomaso E, et al: Lymphatic metastasis in the absence of functional intratumor lymphatics. Science 296: 1883-1886, 2002

39. Cao Y and Zhong W: Tumor-derived lymphangiogenic factors and lymphatic metastasis. Biomed Pharmacother 61: 534-539, 2007.

40. Saito Y, Nakagami H, Morishita R, et al: Transfection of human hepatocyte growth factor gene ameliorates secondary lymphedema via promotion of lymphangiogenesis. Circulation 114: 1177-1184, 2006.

41. Cao R, Ji H, Feng N, et al: Collaborative interplay between FGF-2 and VEGF-C promotes lymphangiogenesis and metastasis. Proc Natl Acad Sci USA 109: 15894-15899, 2012.

42. Cimpean AM, Seclaman E, Ceauşu R, et al: VEGF-A/HGF induce Prox-1 expression in the chick embryo chorioallantoic membrane lymphatic vasculature. Clin Exp Med 10: 169-172, 2010 . 